\title{
Correction to: Environmental factors and wood qualities of African blackwood, Dalbergia melanoxylon, in Tanzanian Miombo natural forest
}

\author{
Kazushi Nakai ${ }^{1,5^{*}}$ (D) Moriyoshi Ishizuka², Seiichi Ohta ${ }^{2}$, Jonas Timothy ${ }^{3}$, Makala Jasper ${ }^{3}$, Njabha M. Lyatura ${ }^{4}$, \\ Victor Shau ${ }^{4}$ and Tsuyoshi Yoshimura ${ }^{5}$
}

\section{Correction to: J Wood Sci (2019) 65:39}

https://doi.org/10.1186/s10086-019-1818-0

In the original publication of the article [1], the scientific name of Mlondondo was misspelled as "Xeoderis stuhlmannii" instead of "Xeroderris stuhlmannii" in Table 1. The corrected Table 1 is given in this correction article.

\section{Author details}

${ }^{1}$ Musical Instruments \& Audio Products Production Unit, Yamaha Corporation, 10-1 Nakazawa-cho, Naka-ku, Hamamatsu 430-8650, Japan. ${ }^{2}$ Japan International Forestry Promotion \& Cooperation Center, Rinyu Building, 1-7-12 Koraku, Bunkyo-ku, Tokyo 112-0004, Japan. ${ }^{3}$ Mpingo Conservation \& Development Initiative, P.O. Box 49, Kilwa Masoko, Kilwa, Lindi, Tanzania. ${ }^{4}$ Kilwa District Council, P.O. Box 160, Kilwa Masoko, Kilwa, Lindi, Tanzania. ${ }^{5}$ Research Institute for Sustainable Humanosphere, Kyoto University, Gokasho, Uji, Kyoto 611-0011, Japan.

Published online: 16 November 2019
Table 1 Local and scientific names of trees in the survey sites $[24,30,36]$

\begin{tabular}{lll}
\hline Local Name & Scientific Name & Family \\
\hline Mpingo & Dalbergia melanoxylon & Fabaceae \\
Mhani & Brachystegia spp. & Fabaceae \\
Miombo & Brachystegia spiciformis & Fabaceae \\
Msolo & Pseudolachnostylis maprouneifolia & Phyllanthaceae \\
Mnepa & Pteleopsis myrtifolia & Combretaceae \\
Mtomoni & Diplorhynchus condylocarpon & Apocynaceae \\
Kingonogo & Combretum spp. & Combretaceae \\
Pangapanga & Millettia stuhlmannii & Fabaceae \\
Mchenga & Julbernardia paniculata & Fabaceae \\
Mtondoro & Julbernardia paniculata & Fabaceae \\
Msumari & Not identified & \\
Msenjele & Acacia nigrescens & Fabaceae \\
Muhungo & Acacia robusta & Fabaceae \\
Mjare & Sterculia appendiculata & Malvaceae \\
Mtandawara & Markhamialutea & Bignoniaceae \\
Mlondondo & Xeroderris stuhlmannii & Fabaceae \\
\hline
\end{tabular}

Some species have not yet been identified. Mchenga in Nainokwe and Mtondoro in Nanjirinji had different local names, but were identified as the same species

Reference

1. Nakai K, Ishizuka M, Ohta S, Timothy J, Jasper M, Lyatura NM, Shau V, Yoshimura T (2019) Environmental factors and wood qualities of African blackwood, Dalbergia melanoxylon, in Tanzanian Miombo natural forest. J Wood Sci 65:39. https://doi.org/10.1186/s10086-019-1818-0

\section{Publisher's Note}

Springer Nature remains neutral with regard to jurisdictional claims in published maps and institutional affiliations. 